Manuelle Medizin 2017 · 55:55

DOI 10.1007/s00337-016-0193-9

Online publiziert: 5. Oktober 2016

๑) Springer-Verlag Berlin Heidelberg 2016

CrossMark

\title{
Christian Holland
}

Emmerich, Deutschland

\section{Vermeintlich gesichertes Wissen}

\section{Originalpublikation}

Mizher A, Rüegg A (2016) Erhebliche

Wirbelsäulenschäden schon durch kleine

Beinlängendifferenzen. Man Med 54:150-155

Sehr geehrter Herr Mizher,

leider fand ich erst jetzt Zeit in das Juniheft zu schauen. Ihr Beitrag „Erhebliche Wirbelsäulenschäden schon durch kleine Beinlängendifferenzen" hat mich sehr erstaunt. Viele Behauptungen werden als vermeintlich gesichertes Wissen dargestellt.

Im Einzelnen nur als Beispiel für mehrere andere: Sie wirken automatisch auf die Kraftübertragung an der Wirbelsäule und verursachen segmentäre Schäden an den Wirbelsäulenabschnitten.

Und was soll ein Satz wie der folgende? Die Muskelfasern sind offensichtlich durch Erbanlagen vorgegeben. Und wie beobachtet man sie genau, analysiert und dokumentiert sie?

Die Röntgenaufnahmen 1-3 bilden jeweils weder beide Beckenkämme noch die Hüftgelenke ab. So muss z. B. eine Beckendrehung doch ausgeschlossen sein, weil dabei bei gleichem Beckenkammstand projektionsbedingt ein kürzeres Bein vorgetäuscht wird - eine solche Beckendrehung liegt bei Aufnahme 2a vor. Bei Aufnahme 3a wird offenbar der asymmetrische Übergangswirbel nicht einmal erwähnt und als mögliche Ursache der Verbiegung diskutiert. Wie kommt der unterschiedliche Stand der Sprunggelenke auf der Aufnahme 4c zustande?

Schon eine leichte Beugestellung von Hüft- und/oder Kniegelenk führt zu einer (scheinbaren) Beinlängendifferenz (BLD), auch eine unterschiedliche Formabweichung an den Füßen oder eine fortschreitende Varusgonarthrose.
Dann entsteht eine BLD erst auch im späteren Lebensalter, in dem schon erhebliche degenerative Veränderungen vorliegen können, wie bei der Patientin in der Abb. 2a und Abb. 2b, bei der man übrigens auch eine Osteoporose vermuten darf! Das soll ein Beispiel für einen BLDbedingten Wirbelsäulenschaden sein?

Die 3 Vergleichsgruppen sind klein, inhomogen und belegen rein gar nichts dienen aber einer Behauptung als Beleg: Es seien damit andere Studien widerlegt! Ist es nicht merkwürdig, dass zwar die Namen der Autoren genannt sind, diese aber im Literaturverzeichnis nicht auftauchen?

Mit kollegialen Grüßen

Prof. Christian Holland

\section{Korrespondenzadresse}

\section{Prof. C. Holland}

Amselweg 11, 46446 Emmerich, Deutschland holland-emmerich@t-online.de

Interessenkonflikt. C. Holland gibt an, dass kein Interessenkonflikt besteht. 